\title{
Design of low-cost wireless noise monitoring sensor unit based on IoT concept
}

\author{
Maja Anachkova $^{1}$, Simona Domazetovska $^{2}$, Zlatko Petreski $^{3}$, Viktor Gavriloski $^{4}$ \\ Ss. Cyril and Methodius University, Faculty of Mechanical Engineering, \\ Skopje, Republic of North Macedonia \\ Institute of Mechanics, Skopje, Republic of North Macedonia \\ ${ }^{1}$ Corresponding author \\ E-mail: ${ }^{1}$ maja.anachkova@mf.edu.mk, ${ }^{2}$ simona.domazetovska@mf.edu.mk, ${ }^{3}$ zlatko.petreski@mf.edu.mk, \\ ${ }^{4}$ viktor.gavriloski@mf.edu.mk
}

Received 25 September 2020; received in revised form 4 January 2021; accepted 18 January 2021 DOI https://doi.org/10.21595/jve.2021.21709

Check for updates

Copyright (C) 2021 Maja Anachkova, et al. This is an open access article distributed under the Creative Commons Attribution License, which permits unrestricted use, distribution, and reproduction in any medium, provided the original work is properly cited.

\begin{abstract}
The recent expansion of wireless sensor networks (WSN) in urban cities has led to possibility of generation of large amounts of information data from the environmental monitoring systems among which one of the most concerning is the excessive urban noise pollution. As opposed to the conventional noise mapping procedures that involve costly and time-consuming measurement process with a traditional high-priced noise level meter, the low-cost wireless sensor networks provide a method for achieving data collection and analysis with a higher level of granularity. This paper presents a wireless noise sensor unit design for continuous environmental noise level monitoring as a framework for the realization of the Internet of Things (IoT) and „Smart city" concept. The concept involves the complete noise data information system, from sensor structure to data visualization and data analysis. The overall design, characteristics and performance of the sensing system for continuous measuring urban noise pollution is discussed.
\end{abstract}

Keywords: urban noise monitoring, low-cost noise sensor, internet of things (IoT), smart city concept.

\section{Introduction}

As a consequence of the intensive centralization of large cities, urban settlements are beginning to expand, reaching extensive levels of noise coming from various sources such as human leisure activities, traffic, construction activities and industry that result in residents' annoyance and health problems. Conventional noise measurements in urban areas are mainly carried out by professionals that record and analyze noise data at a location of interest using a hand-held sound level meter. Such a manual and laborious method does not respond well on the demand for more noise measurements in time (longer periods), space (more locations) and cost (expensive sound level meter). Therefore, producing accurate and meaningful noise maps based on the conventional procedure needs to be facilitated.

The last decade has shown a growing number and wide variety of systems for monitoring environmental noise that can help in getting more insight in often complex noise situations in less time and labor. In these terms, custom built sensor network solutions are designed to be small, inexpensive, low power and autonomous so that it can be deployed pervasively, enabling monitoring in a wide area [1]. With the popularization of IoT, this topic is becoming more and more relevant and aims to monitor the level of noise in cities by designing and developing (WASNs - Wireless Acoustic Sensor Networks). In reality, the idea of Internet of Things is established by the communication of sensors and electronic devices capable of offering low price monitoring at the side of a growing infrastructure, which allows observing tasks with a reduced cost. According to some industry analysis [2], in 2015 there were between 10 and 20 billion monitoring units or objects connected to the Internet and it is generally estimated that this number achieves $40-50$ billion in 2020 .

The multifarious opportunities that arise from the IoT-enabled monitoring and control 
capabilities have motivated a greater number of researchers towards investigating the innovative IoT solutions for noise monitoring and control in urban environments. The projects in [3-5] deploy a network of low-cost commercial sound level meters, with ZigBee technology and GPRS communication for data gathering, with the final goal of collecting the equivalent noise level in several parts of the city. This is also the ultimate aim of the DYNAMAP project, which describes sensor units that achieve good accuracy for least cost deployed in two pilot areas in Italy [6]. The sensor nodes designed for that network are low-cost and use Class 2 MEMS microphones. The IoT concept solutions for noise measurement sensor networks can be constructed differently, meaning different noise sensors, controllers, transmission of the acquired data, cloud storage service or user applications can be used. For example, in [7], a distributed noise measurement system based on IoT technology is developed with Raspberry Pi controller nodes and a data collection system based in OpenCPU framework. This system has been tested to measure the hourly sound pressure level evolution. In paper [8] is presented a model that uses microcontroller ATMEGA328 that is mounted on Arduino Uno board to measure the fluctuations in noise levels with high sensitivity microphone sensor module. The goal of the proposed methodology is to design and develop a technique that can lead towards a cost-effective and scalable traffic noise management system. In order to achieve re-usability and heterogeneity, VITAL-OS2, an open-source IoT platform for Smart Cities has been performed in [9]. The paper [10] presents the implementation of a sound-level metering IoT system based on MQTT protocol. The implemented system has been created by using Raspberry Pi 3, Arduino MEGA, ESP8266 Wi-Fi module and sound pressure level meter. The collected data can be stored in database created on some server for further processing to obtain relevant statistical parameters of environmental noise. In paper [11], the sensing system is built on Atmega128 and CC2420 platform. The protocol stack is based on Ci-Net stack. Strict filtering function specified by ITU-R 468 (namely A-weighting) is followed. Both the indoor and outdoor test results were compared with a standard sound level meter and showed less than $\pm 2.1 \mathrm{~dB}$ mean differences in both short-term and long-term measurement. In [12], the nodes are composed by a microcontroller ATmega 328, arranged in a board model Stalker v.3 from Seeedstudio, a Wi-Fi transmitter model ESP8266 from Espressif Systems Inc. It also has a board called Sensor Shield V2 that connects directly on the board Stalker, so to facilitate the connection of the sensors [13]. In study [14], a wireless sensing unit (WSU) that has the same functionality as a handheld sound level meter is exposed. The WSU consists of a microcontroller unit that enables on-board computations, a wireless transceiver that uses Zigbee protocol for data transmission, and an external peripheral board with a microphone transducer.

Inspired by the previous scientific studies, a low-budget, self-powered and wireless sensor unit for continuous collection and visualization of data for the environmental noise level is developed. The concept design of the monitoring device is explained in Section 2. The device design from a constructive point of view is presented in Section 3 and further in Section 4, the calibration in indoor (laboratory) and outdoor conditions is explained. In Section 5 are presented the results collected from the sensor for the Leq (equivalent sound level) parameter on a location on the campus of the Faculty of Mechanical Engineering in Skopje on a daily, as well as hourly basis time averaged. In Section 6 are discussed the future plans and possibilities in terms of deployment and expansion of the sensor network. At the end, conclusions are exposed in Section 7.

\section{Concept design and development}

The IoT technology concept is being used for continuous monitoring of many dynamic parameters in the environment monitoring among which is the noise pollution. The main aspect of this technology is to replace the expensive measuring devices with cheaper sensors units, which will provide accurate results and simplify the monitoring process. This concept aims through precisely established methods of optimization and calibration, from a low-cost sound measuring sensor that has limited performance, to provide a sound measuring sensor whose accuracy can be 
competitive to a professional Class I sound level meter. Furthermore, this node can be replicated to provide a small network of sensors through which information about the noise level in a wider area will be possible to obtain. Such sensor unit can be used to monitor and analyze noise through sensors placed at multiple locations throughout the city in order to present and visualize the noise level situation in the area while the processed data would be made available, via maps, to the general public. Consequently, it will be possible to monitor the contemporary level of noise in order to increase awareness of the ubiquity of noise in the lives of citizens due to the specific invisible nature of the problem.

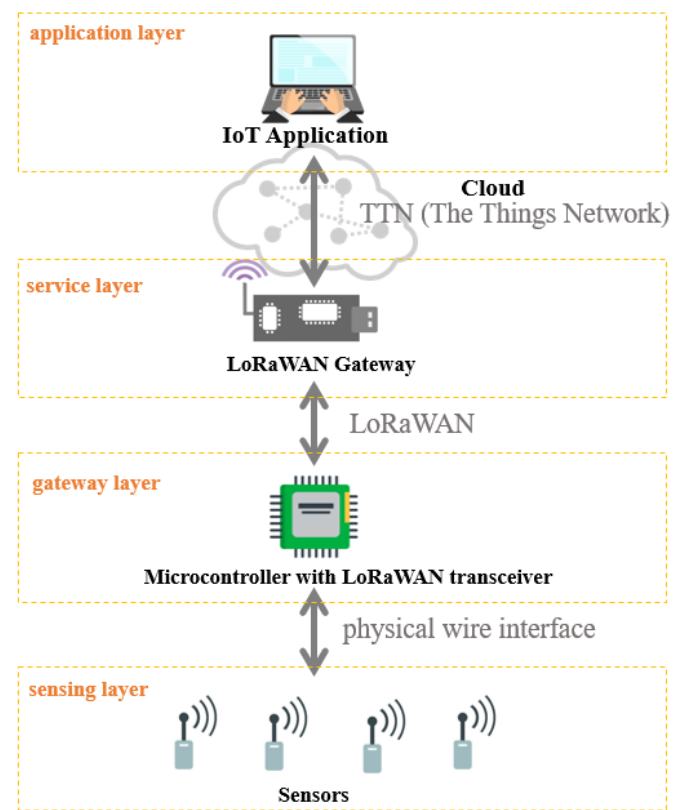

Fig. 1. Four main layers of the monitoring system

Generally, every IoT monitoring system must be consisted of four layers as given in Fig. 1 [15]. Namely, the first layer is the sensing layer which consists of noise sensor (microphone) for receiving the noise level information and the microcontroller. Second is the gateway layer that is a local gateway device which is responsible for authentication of sensor nodes and transmission of encrypted data and this layer consists of connectivity components and provides the Internet connectivity to the system. The service layer as third, consists of a cloud server and data storage, while the application layer provides the interface to the system. End users can monitor the obtained results and control the system on the user interface. As can be concluded from the literature review, all four layers of the system can be developed using different types of components, protocols and platforms, although they all serve for the final purpose of noise level monitoring. In this research, the sensor device is connected to the LoRaWAN network, which enables connection to a chosen web platform for data analysis. Further aspect is the visualizations of the results, for which the ThingSpeak, a network server for IoT infrastructure that uses LoRaWAN as a core technology is used.

\section{Design of the sensor unit}

The noise measuring system having the task of continuous data collection for the outdoor noise levels is constructed of a microprocessor, microphone, battery and information transfer module. Namely, in this case a Grove sensor is used as a transducer, Arduino Uno microcontroller as a sensing layer, Lo-Ra click module as a gateway layer for information transmission, TTN as a 
service layer for cloud storage and the ThingSpeak platform as an application layer. The main component of the Grove sensor is the microphone, which is based on the electrode (permanently polarized piece of dielectric material) and LM358 amplifier. The sensitivity of the microphone at $1 \mathrm{kHz}$ is $52-48 \mathrm{~dB}$ and its' measurement range is from $42 \mathrm{~dB}$ to $90 \mathrm{~dB}$ which is acceptable in the range for the outdoor noise levels. The information detected from the noise sensor is processed by the Arduino Uno microcontroller and afterwards sent via the Lo-Ra click module and the antenna to the cloud of the global TTN platform where it is stored. The complete design with the consisting components of the sensor unit is shown in Fig. 2. The basic principle of data collection and visualization process is as follows: the device collects information about the noise level through the microphone, which are processed by the microcontroller, sent and further stored to TTN cloud in order to be numerically and graphically visualized on the server by the ThingSpeak platform. Under these terms, the device is wireless, self-powered and connected to a global Internet network.

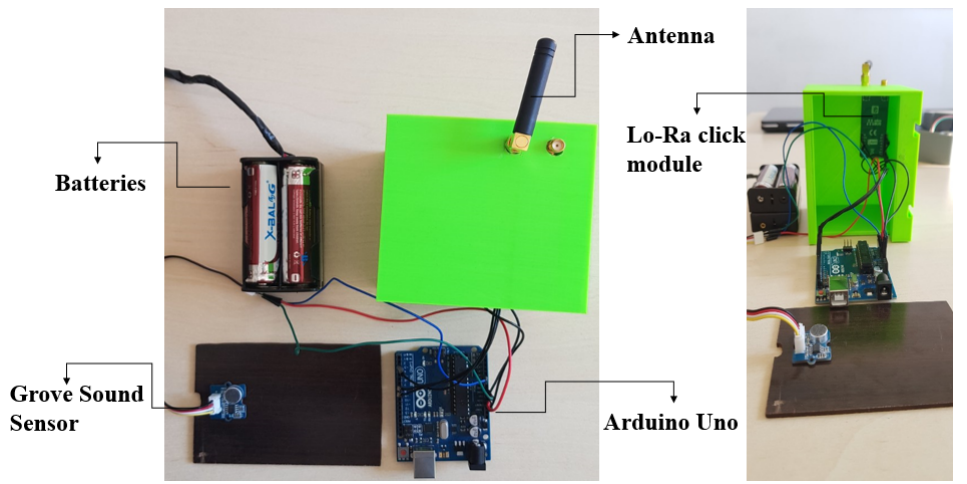

Fig. 2. Elements of the sensor node

In order to assure safe outdoor operation of the electrical components of the sensor node, the sensor box is designed with ABS plastic which has high resistance to corrosive chemicals and physical impacts. Due to the relatively low price $(1.87 € / \mathrm{kg})$ and easy processing, ABS plastic is used for 3D printing. The sensor itself is placed in the box by sliding between the slots at the end of the box, so that the microphone is faced outwards. According to the standards for outdoor environmental noise measurements, in the case of a reflective surface, the microphone should be placed at least 3 meters from the surface. If the microphone is placed on a reflecting surface, the measured noise level should be corrected to $-6 \mathrm{~dB}$. at a height of $1.5 \pm 0.1 \mathrm{~m}$ above the base.

In order to be easily replicated for deployment of a larger sensor network and fulfill the expectations of the IoT concept itself, the sensor unit was constructed by using affordable low-cost components. The costs for the unit are calculated to be around $90 €$ and the prices for each component are given in Table 1. Compared to the sound level meters used in the conventional noise measurements which can cost a couple of thousands of Euros (over $€ 1000$ ), this unit is very moderately priced [11].

Table 1. Components of the sensor unit

\begin{tabular}{|c|c|}
\hline Components & Price \\
\hline Grove sound sensor & $5 €$ \\
\hline Arduino Uno Rev3 Microcontroller & $22 €$ \\
\hline Lo-Ra click module & $54 €$ \\
\hline Battery & $2 €$ \\
\hline Antenna & $7 €$ \\
\hline Total & $90 €$ \\
\hline
\end{tabular}

Nevertheless, cheap measurement hardware cannot promise high-accuracy and the measurement error is not technically determined. Therefore, it was decided to perform a 
calibration procedure of the sensor with a professional Class 1 Bruel\&Kjaer sound level meter.

\section{Sensor node calibration}

The calibration of the Grove sound sensor with a Class 1 sound level meter by Bruel\&Kjaer was performed in laboratory indoor conditions using audio system, at 13 different frequencies at a same amplitude of an amplifier.

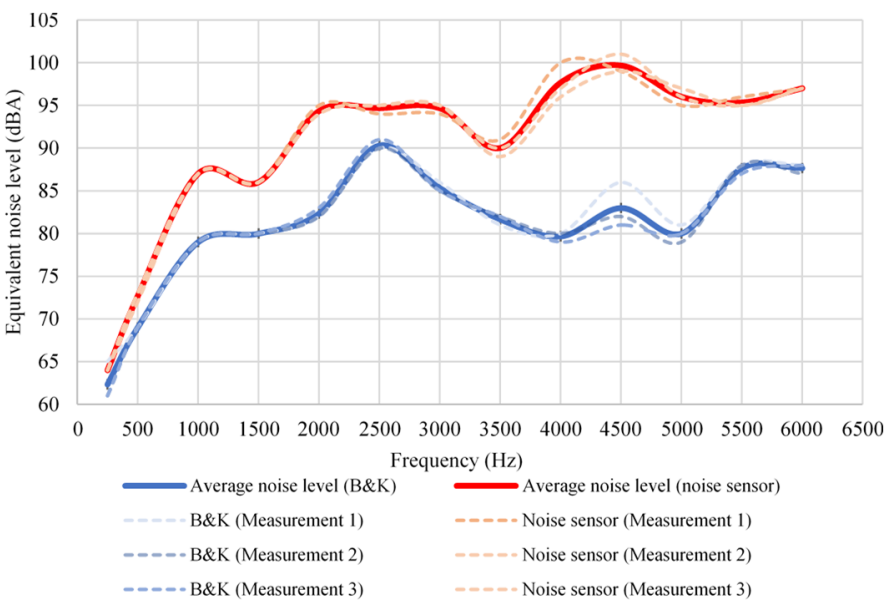

Fig. 3. Laboratory calibration results

Both instruments, the Bruel\&Kjaer Class 1 sound level meter and the Sound Grove sensor were installed under same conditions, at the same distance and height from the speaker. In order to ensure repeatability of the results, the measurements of the sound levels were repeated 3 times at each frequency and the curves of the obtained results are shown in Fig. 3. The blue curves interpolate and display the average values for the sound level measured at the different frequencies with the Bruel\&Kjaer Class 1 sound level meter, while the red curves show the average sound level values measured with the sensor. The curves shown by the dashed lines are the values obtained in each of the three measurements made, while the filled bold lines are the values obtained by averaging the results of each of these three measurements. From the diagram can be concluded that different discrepancies between the measured sound levels from the two instruments occur at different frequencies. Namely, larger differences are detected at higher frequencies above $3.5 \mathrm{kHz}$, especially on $4.5 \mathrm{kHz}$, while at lower frequencies the error of the noise sensor in comparison with the sound level meter is smaller. It can be seen that in the range of $500 \mathrm{~Hz}$ to $3 \mathrm{kHz}$, the value read out from the low-cost noise sensor ranges from at least 2 to at most $12 \mathrm{~dB}$. The calibration has shown a significant difference in the results, which leads to the conclusion that a correction in the code is necessary to be made in order to provide a more equal response from the sensor.

After the code in Arduino was modified, in order to re-examine the sensor performance, the calibration was re-performed, this time in an outdoor environment. The sensor and the sound level meter were placed in the same location and under the same external conditions for a period of five hours (from $10 \mathrm{AM}$ to $3 \mathrm{PM}$ ), where the obtained values for Leq parameter can be seen in Fig. 4. The microcontroller was programmed to calculate the Leq value for every 10 minutes time interval. A time interval of 10 minutes was chosen as a minimum interval required for determination of Leq according to the Directive 2002/49/EC and the standards included. The sensor is set for a sampling time of $20 \mathrm{~s}$, which means that the Leq value for the time slot of 10 minutes is calculated out of 30 samples (values) for the sound level.

From the graph in Fig. 4 can be noticed that the trend of the curves appears to be more sustained 
after the corrections of the code. On the other hand, the deviations of $2 \mathrm{~dB}$ in Leq values for higher values of the sound levels from 10:20 to 10:40 and from 11:00 to 11:40 are obvious, whereas the values obtained from the sensor deviate up to a maximum of $3 \mathrm{~dB}$ at 10:20 and 11:20. Also, a larger difference of about $4 \mathrm{~dB}$ can be noticed in the period from 12:30 to 13:10. The general conclusion from the comparison of the sound level meter and noise level sensor is that at higher sound levels (above $60 \mathrm{~dB}$ ) there is a noticeably larger error in the measured values, while for the lower sound levels the measured values almost overlap.

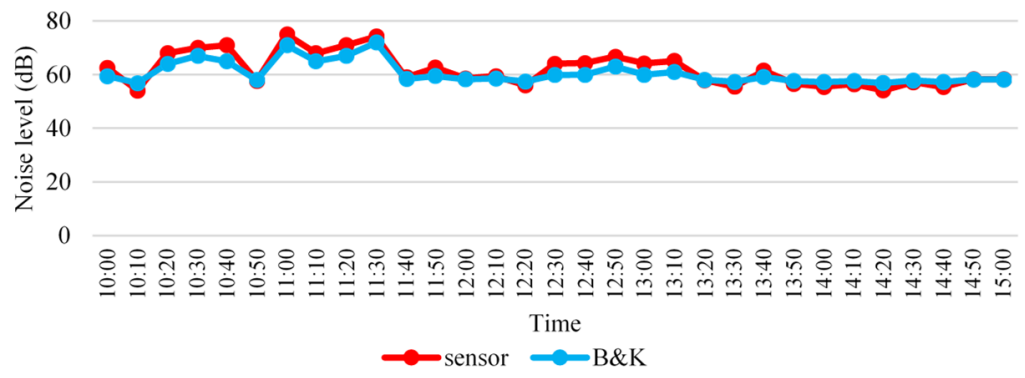

Fig. 4. Outdoor calibration results

\section{Results}

After calibration processes have provided the acceptable results, the module was placed on a chosen location on the Faculty campus as shown in the photograph in Fig. 5.

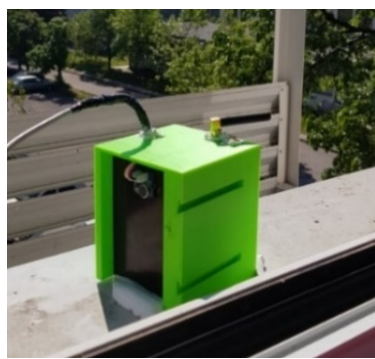

Fig. 5. Sensor unit placed on the campus

The sensor was pre-set to give a value for the Leq calculated for a period sample of 10 minutes and throughout 30 days, the measured values were monitored on the created ThingSpeak channel. The graph shown on the Fig. 6(a), shows the obtained results for the daily Leq values from the sensor, while the graph on Fig. 6(b) provides the results for the Leq gathered during an arbitrarily chosen period of a day. In the city of Skopje are installed three Class A LoRaWAN stations. The coverage of the LoRaWAN gateway is approximately $4-5 \mathrm{~km}$ in radius, nevertheless it is also dependent on the spreading factor (SF) used which in this case is $\mathrm{SF}=10$. The frequency is set to be EU868,3 MHz with a duty-cycle of $1 \%$ with max payload (byte) of 51 and max application data (byte/h) of 2601 with a coding rate of 4/5. Due to the low speed of $980 \mathrm{bits} / \mathrm{s}$ and the bandwidth of BW=125 kHz, the airtime estimated on The Thing Network platform is 206,878 seconds.

From the presented measured results on the ThingSpeak platform obtained from the sensor unit during this period (Fig. 6), it can generally be noticed that the location where the sensor is placed provided a stable connection and good transmission of data with the LoraWAN emitter, i.e there is no communication interruption between the emitter and LoRa click module installed in the unit. The energy consumption depends directly from the SF, as well as the payload size which is in small packages 1-20 bytes (2-200 uplinks per day and 1-10 downlinks per day, one uplink is 
followed by 2 downlinks at 1 s period), number of packets sent per day which in this case is 144 ( 24 hours $\times 6$ packets/hour). The sensor was powered with two $1.5 \mathrm{~V}$ batteries that in this case for the initial pilot testing endured through a period of two weeks. During this one-month trial period, the sensor unit has shown a good sustainability and acceptable performance in terms of acquisition, transmission and representation of the measured values for the noise level.

Another aspect that can be commented in terms of using the ThingSpeak platform, is that it offers a customized approach to displaying graphically representative results. This platform is intended to be used from the general public and is aimed to be easily manipulated and used to fit in the "Smart city" concept. For further analysis, it also offers the option of processing the measured data in MATLAB in order to make more detailed data analysis if needed.

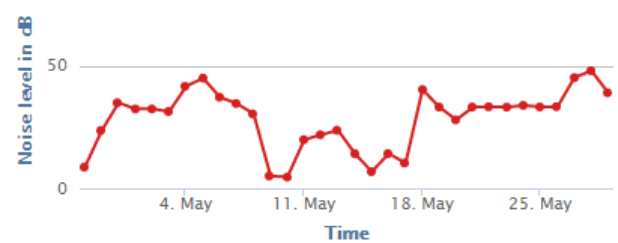

a)

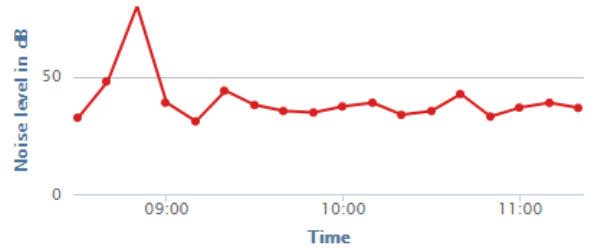

b)

Fig. 6. Visualization of the measured noise values on the ThingSpeak platform: a) daily averaged noise level, b) hourly averaged noise level

\section{Discussion and future work}

In this paper, the main focus is put on the configuration and design of the low-cost wireless sensor unit, its' components and connection to the global Internet network, as well as final visualization of the measured results for the sound level as a main parameter of interest. In parallel, an accent is put on the calibration of the sound sensor in order to ensure the reliability and accuracy of the results. Consequently, another aspect that deserves to be taken into consideration is the next phase after the establishment of the sensor unit which is the multiplication of the sensor unit throughout the campus of the Faculty of Mechanical Engineering in Skopje as a pilot area. This is planned be done in terms of testing the sustainability, durability and accuracy of the sensor over a long period of time. This can enable insight to conclusions about the applicability of such sensor on a larger scale (the urban area of the city where the coverage of LoRaWAN is available), as well as the requirements and expectations that can be achieved by this low-budget wireless sensor unit. The planned distribution of the sensor network can be visualized in Fig. 7.

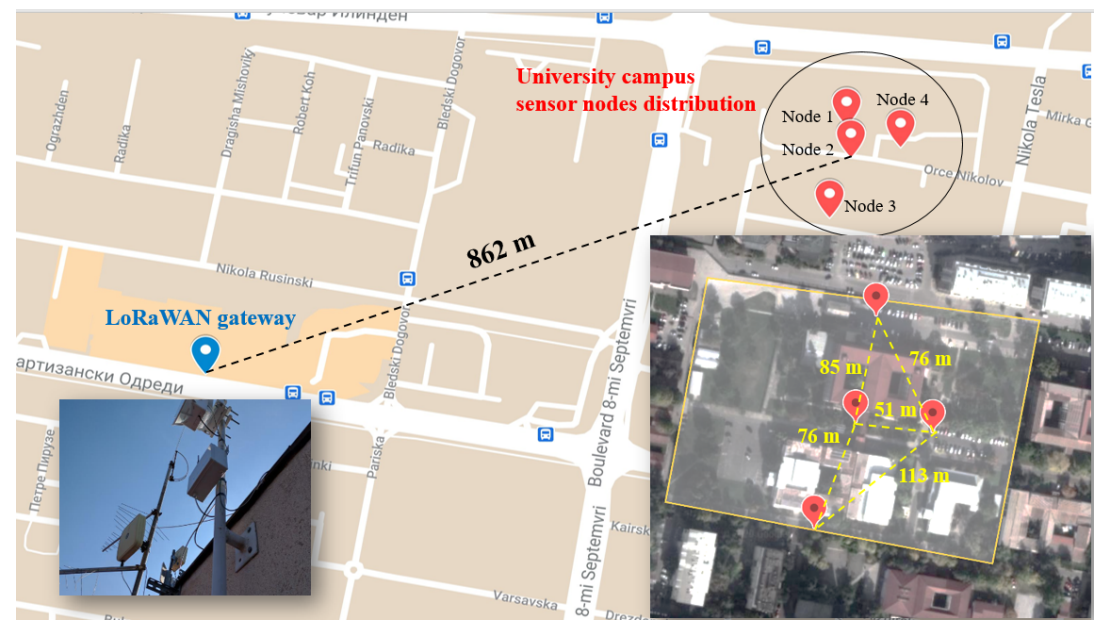

Fig. 7. Sensor unit distribution in the pilot area on the campus of the Faculty of Mechanical Engineering 
The sensors are going to be placed on a few characteristic points on the campus. Namely, the first point will be on the outer facade of the reading room of the Faculty of Mechanical Engineering. The reading room is a place were keeping quiet is crucial. On the other hand, the reading room is located next to the entrance to the parking lot of the faculty, which means that this part of the campus is prone to noise caused by cars passing by. Another sensor will be placed on the outside, above the entrance to the Faculty. This part of the campus is more isolated from the outside traffic. However, this entry is most used by students and professors, so it is exposed to conversation and leisure activities noise. The third point is on the side of the building, which is next to the park, which is a quiet area that students are using for outdoor readings and walks. The last point will be the part of the campus that is right next to a street that has a high traffic frequency during the whole day. The installation of such sensor network covers all four sides of the campus, as well as characteristic points from a noise density and intensity point of view.

\section{Conclusions}

In this paper, a low-budget smart sensor unit for environmental noise level measurement, inspired by the IoT concept is presented. The unit is constructed of a microphone, Arduino Uno microcontroller, Lo-Ra click module and antenna for connection and cloud storage to the global TTN platform. In general, can be concluded that the system has shown good performance in terms of sustainability, acquisition, transmission and representation of the measured values for the noise level. As a main advantage compared to other sensors developed and described in the reviewed state-of-the-art literature is considered the sensor cheapness (other sensors that are considered "low-cost" can reach to $1000 €)$. Also, the sensor node is easy to construct which makes it uncomplicated to replicate and increases the possibility for extension of the sensor network. It easy to connect to LoRAWAN network if a LoRAWAN station is available and the interface is used-friendly and easily manipulated even for non-technicians because the TTN (the Things Network) is an open-access, global and crowd sourced IoT network with possibilities for public use. The system can be safely standalone for at least 2 weeks with the proposed batteries due to the compactly designed $3 \mathrm{D}$ printed housing that is resistive to outdoor conditions. Besides the low-cost of the sensor, it shows good performance in measurement correctness and accuracy with a maximum error of $5 \mathrm{~dB}$ as shown in the paper.

The ultimate goal of the project is to provide a larger scale dense network of identical sensors for continuous monitoring of the sound level values in the city. Visualizing the results for the noise changes will contribute to a better understanding of the noise situation on a particular location and help with the development of the "Smart city" concept in the city of Skopje.

\section{Acknowledgement}

We would like to thank our team of students for the assistance and contribution on the implementation of the presented work.

\section{References}

[1] Basten T. G. H., Wessels P. W. An overview of sensor networks for environmental noise monitoring. Proceedings of the 21st International Congress on Sound and Vibration, Beijing, China, 2014.

[2] Rus C., Negru N., Patrascoiu N. Low-cost system to acquire environmental parameters in urban areas in the context of IOT. Journal of Environmental Protection and Ecology, Vol. 20, Issue 3, 2019, p. 1451-1461.

[3] Wang C., Chen G., Dong R., Wang H. Traffic noise monitoring and simulation research in Xiamen City based on the Environmental Internet of Things. International Journal of Sustainable Development and World Ecology, Vol. 20, Issue 2013, 3, p. 248-253.

[4] Botteldooren D., De Coensel B., Oldoni D., Van Renterghem T., Dauwe S. Sound monitoring networks new style. Breaking New Ground: Annual Conference of the Australian Acoustical Society, 2011. 
[5] Bell M. C., Galatioto F. Novel wireless pervasive sensor network to improve the understanding of noise in street canyons. Applied Acoustics, Vol. 74, Issue 1, 2013, p. 169-180.

[6] Bellucci P., Cruciani F. R. Implementing the dynamap system in the suburban area of Rome. InterNoise and Noise-Con Congress and Conference Proceedings, Vol. 253, Issue 3, 2016, p. 5518-5529.

[7] Segura Garcia J., Perez Solano J. J., Cobos Serrano M., Navarro Camba E. A., Felici Castell S., Soriano Asensi A., Montes Suay F. Spatial statistical analysis of urban noise data from a WASN gathered by an IoT system: application to a small city. Applied Sciences, Vol. 12, 2016, p. 380.

[8] Shukla K., Barot J. A large-scale spreaded sensing system for road conditions, air and sound effluence. International Journal of Research and Analytical Reviews, Vol. 6, Issue 2, 2019, p. 78-79.

[9] Kazmi A., Tragos E., Serrano M. Underpinning IoT for road traffic noise management in smart cities. IEEE International Conference on Pervasive Computing and Communications Workshops, 2018, p. 765-769.

[10] Lekić M., Galić J., Matić S. An IOT solution for secured and remote sound level monitoring. IEEE 18th International Symposium INFOTEH-JAHORINA, 2019.

[11] Kivelä I., Gao C., Luomala J., Ihalainen J., Hakala I. Design of networked low-cost wireless noise measurement sensors. International Journal on Sensors and Transducers, Vol. 9, 2010, p. 171-190.

[12] Sani A. B. B. A., Beauty P. E. Smart framework for environmental pollution monitoring and control system using IoT-based technology. Sensors and Transducers, Vol. 229, 2019, p. 84-93.

[13] Velásquez P., Vásquez L., Correa C., Rivera D. A low-cost IoT based environmental monitoring system. A citizen approach to pollution awareness. Conference on Electrical, Electronics Engineering, Information and Communication Technologies, 2017.

[14] Peckens C., Porter C., Rink T. Wireless sensor networks for long-term monitoring of urban noise. Sensors, Vol. 18, Issue 9, 2018, p. 3161.

[15] Malche T., Maheshwary P., Kumar R. Environmental monitoring system for smart city based on secure internet of things (IoT) architecture. Wireless Personal Communications, Vol. 107, Issue 4, 2019, p. 2143-2172.

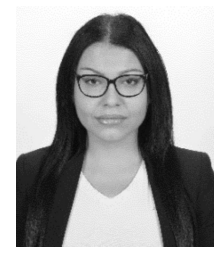

Maja Anachkova is a Ph.D. student at the Faculty of Mechanical Engineering in Skopje, where she currently works as a teaching assistant at the Institute of Mechanics. Her main research area is dedicated to the field of noise and vibration, and the wider field of research includes system mechanics, system dynamics and mechatronics.

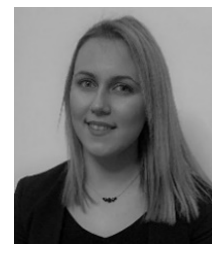

Simona Domazetovska is a Ph.D. student at the Faculty of Mechanical Engineering in Skopje, where she currently works as a teaching assistant at the Institute of Mechanics. Her main research activity in doctoral studies is in the field of environmental noise, and the wider field of research includes mechatronics, mechanics, dynamics and vibration of dynamic machine systems.

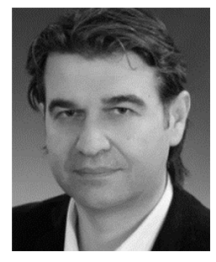

Zlatko Petreski works as a professor at the Institute of Mechanics and mechatronics on the Faculty of Mechanical Engineering, University "Ss. Cyril and Methodius" in Skopje. His current research includes strength of materials, mechanics, noise and vibration analysis and protection, measurement technics and signal processing.

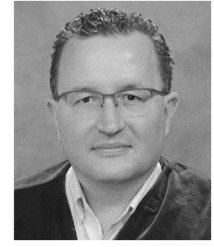

Viktor Gavriloski is a professor at the Institute of Mechanics and mechatronics on the Faculty of Mechanical Engineering, University "Ss. Cyril and Methodius" in Skopje. His field of research interests includes strength of materials, mechanics, noise and vibration analysis and mechatronic systems. 